\title{
Pharmacokinetics of mitragynine in man
}

This article was published in the following Dove Press journal:

Drug Design, Development and Therapy

29 April 2015

Number of times this article has been viewed

\author{
Satariya Trakulsrichai ${ }^{1,2}$ \\ Korbtham Sathirakul ${ }^{3,4}$ \\ Saranya Auparakkitanon ${ }^{5}$ \\ Jatupon Krongvorakul ${ }^{5}$ \\ Jetjamnong Sueajai ${ }^{5}$ \\ Nantida Noumjad ${ }^{5}$ \\ Chonlaphat Sukasem ${ }^{5}$ \\ Winai Wananukul ${ }^{2,6}$ \\ 'Department of Emergency Medicine, \\ Faculty of Medicine Ramathibodi \\ Hospital, ${ }^{2}$ Ramathibodi Poison Center, \\ Faculty of Medicine Ramathibodi \\ Hospital, ${ }^{3}$ Department of Pharmacy, \\ Faculty of Pharmacy, Mahidol \\ University, Bangkok, Thailand; ${ }^{4}$ Center \\ for Drug Research Discovery and \\ Development, Thammasat Univerisity, \\ Prathumthani, Thailand; ${ }^{5}$ Department \\ of Pathology, Faculty of Medicine \\ Ramathibodi Hospital, ${ }^{6}$ Department \\ of Medicine, Faculty of Medicine \\ Ramathibodi Hospital, Mahidol \\ University, Bangkok, Thailand
}

Correspondence: Winai Wananukul Ramathibodi Poison Center and Department of Medicine, Faculty of Medicine Ramathibodi Hospital, Mahidol University, 270 Rama VI Road, Ratchatewi, Bangkok 10400, Thailand Tel +66 220 I 1084

Fax +66 220I 1086

Email winai.wan@mahidol.ac.th
Background: Kratom, known botanically as Mitragyna speciosa (Korth.), is an indigenous tree in Southeast Asia. Kratom is currently easily available worldwide via special shops and the Internet to use as a drug of abuse, opioid alternative, or pain killer. So far, the pharmacokinetics of this plant has been studied only in animals, and there is no such study in humans. The major abundant active alkaloid in Kratom, mitragynine, is one of the promising new chemical substances to be developed as a new drug. The aim of this study was to examine the pharmacokinetics of mitragynine and assess the linearity in pharmacokinetics in chronic users.

Methods: Since Kratom is illegal in Thailand, studies in healthy subjects would be unethical. We therefore conducted a prospective study by enrolling ten chronic, regular, healthy users. We adjusted the steady state in each subject by giving a known amount of Kratom tea for 7 days before commencement of the experiment. We admitted and gave different oral doses to subjects to confirm linearity in pharmacokinetics. The mitragynine blood concentrations at 17 times points and the urine concentrations during the 24-hour period were collected and measured by liquid chromatography-tandem mass spectrometry method.

Results: Ten male subjects completed the study without adverse reactions. The median duration of abuse was 1.75 years. We analyzed one subject separately due to the abnormal behavior of blood concentration. From data of nine subjects, the pharmacokinetic parameters established were time to reach the maximum plasma concentration $(0.83 \pm 0.35$ hour $)$, terminal half-life (23.24 \pm 16.07 hours), and the apparent volume of distribution $(38.04 \pm 24.32 \mathrm{~L} / \mathrm{kg})$. The urine excretion of unchanged form was $0.14 \%$. The pharmacokinetics were observed to be oral twocompartment model.

Conclusion: This was the first pharmacokinetic study in humans, which demonstrated linearity and was consistent with the oral two-compartment model with a terminal half-life of about 1 day. The pharmacokinetic linearity and parameters reported are necessary pharmacological information of Kratom, and there is a possibility for it to be developed medically as a pain killer or better opioid substitute in the future.

Keywords: kratom, human, pharmacokinetics

\section{Introduction}

Kratom, the botanical name of Mitragyna speciosa (Korth.), also known as Ketum in Malaysia, belongs to the Rubiaceae family, which can grow in tropical and subtropical regions of Asia. ${ }^{1,2}$ It is a native tree indigenous to Southeast Asia, especially in Thailand and Malaysia., ${ }^{2,3}$ It contains many alkaloids such as mitragynine, paynantheine, speciogynine, and 7-hydroxymitragynine. ${ }^{2}$ Mitragynine is the most abundant active alkaloid isolated from Kratom., ${ }^{2,4}$ Mitragynine has an antinociceptive effect, similar to 7-hydroxymitragynine, one of alkaloids, which is a significantly more potent analgesic than mitragynine and morphine. ${ }^{2,4}$ Kratom possesses dose-dependent pharmacological effects, with stimulant effects in lower doses and opiate-like effects in higher doses. ${ }^{4}$ Other clinical effects include antinociceptive, antiinflammatory, antidiarrheal, antitussive, antipyretic, euphoric, antidepressant, anxiolytic, blood pressure 
lowering, antidiabetic, and appetite suppressing as previously described. ${ }^{2,4}$ Kratom is also reported to be used as an opioid substitute. $^{5-7}$

There were studies performed to observe the effect of mitragynine several years ago. ${ }^{8,9}$ The acute toxicities studied in rats include increasing blood pressure, hepatotoxicity, and nephrotoxicity. ${ }^{10}$ In another rat study, it was found that the aqueous extract, even very high dose, did not cause death and any significant toxicity. ${ }^{11}$ In humans, the adverse effects or the toxicities include dry mouth, changes in urination, nausea, vomiting, anorexia, weight loss, constipation, nystagmus, and tremor. ${ }^{4,12}$ In addition, seizure has been reported in previous literature. ${ }^{5,13,14}$ Primary hypothyroidism ${ }^{15}$ and intrahepatic cholestasis $^{16}$ are also reported in a number of cases to be associated with Kratom. For chronic toxicity, impairment of cognitive behavioral function is found in mice fed mitragynine for a long period. ${ }^{17}$ In humans, anorexia, weight loss, hyperpigmentation, and psychosis are described in chronic abusers. ${ }^{4}$ Kratom alone, or in combination with other substances, has been shown to cause dependence and withdrawal symptoms following repeated consumption. ${ }^{2,18-21}$ Kratom has the potential herb-drug interaction on cytochrome P450 (CYP) enzyme activity. This was shown in one study, with potent inhibitory effect for CYP3A4 and CYP2D6, moderate effect for CYP1A2, and weak effect for CYP2C19, ${ }^{22}$ whereas in another study, mitragynine and 7-hydroxymitragynine also showed the inhibitory effect on P-glycoprotein. ${ }^{23}$ However, there were a few reports of Kratom-related fatalities. ${ }^{24,25}$

In Thailand, Kratom is used by manual laborers to enhance physical endurance and productivity and to elicit euphoric effects. It is also used as a folk medicine to treat several diseases or in the past as an opioid alternative by chewing fresh leaves, smoking dried leaves, or brewing as a tea. ${ }^{2-4,12}$ In the south of Thailand, Kratom is not considered to be a drug of abuse, and it is a part of the way of life, embedded in local custom and tradition. Nevertheless, now it is causing concern, emerging as drug of abuse known as " $4 \times 100$ cocktail" and one of the most popular illicit substances used, 2,14 particularly in the southern part of Thailand. Although, Kratom, a schedule 5 drug in Thai Narcotic Act, is banned in some countries such as Malaysia, it is still legal in Indonesia and the United States and some countries in Europe. ${ }^{2}$

Kratom has become increasingly trendy in several countries, especially in Europe and USA. Currently, it is available through special shops or online market known as "Legal highs." Therefore, it is used by the online community and made available by many online vendors. ${ }^{2}$ It is important to note that Kratom is sometimes used as a drug of abuse alone or in combination with other substances and for medicinal purposes such as to relieve pain., ${ }^{2,426-34} \mathrm{Kra}-$ tom is used to alleviate opioid withdrawal symptoms and is an economical alternative to other opioid-replacement medications without a prescription. There are an increasing number of reports, studies, and reviews about Kratom use for opioid substitution. ${ }^{2,5-7,35}$ Despite increasing reports and studies on Kratom, to our knowledge, respiratory depression or significant opioid toxic syndrome have not been reported as the toxicity from Kratom. ${ }^{7,14,35}$ Even if Kratom is mainly available as a drug of abuse, it may yet provide insight into the possibility for its medicinal development as a new and more effective opioid substitute or pain killer in the future, with fewer lethal side effects.

To date, current knowledge of Kratom is limited, and many questions remain regarding its basic safety and the potential toxicities.

Hence, the pharmacology and pharmacokinetics are very important and necessary data to understand in managing poisoning or even widening the medical scope of Kratom in the future. Currently, the pharmacokinetics of Kratom has been derived only from animal studies, ${ }^{36-38}$ which employed only the rat model and produced variable results. There is no pharmacokinetic study in humans. Thus, the objective of this study was to look for the first time at the pharmacokinetics in humans and to assess the linearity of the pharmacokinetics of mitragynine, the most prevalent alkaloid in Kratom. This active alkaloid is a promising new chemical for new drug development.

\section{Methods}

\section{Study design and subjects}

The study was approved by Institutional Review Board of the Faculty of Medicine, Ramathibodi Hospital, Mahidol University. Since Kratom is an illegal substance in Thailand, performing the study in healthy subjects is unethical; therefore, we performed the prospective experimental study in chronic regular users. We asked for permission to possess Kratom leaves from the Thai Food and Drug Administration to be used in this study.

The primary outcome was the pharmacokinetics of mitragynine. The secondary outcomes were blood pressure and pulse rate change after taking Kratom. We used different doses of Kratom to observe the dose responses in our study.

Inclusion criteria for recruiting subjects in this study included healthy participants, without any underlying diseases, using Kratom regularly for more than 6 months, having positive urine or blood test for mitragynine at the first hospital visit. We excluded subjects who refused to participate in the study. All subjects provided written informed consents. 


\section{Study protocol}

\section{Preparation of Kratom tea}

Kratom tea for this study was prepared from leaves collected from Phatumthani province, Thailand during 2012-2013. Ramathibodi Poison Center, Faculty of Medicine Ramathibodi Hospital was certified to handle the plant material. First, fresh or dried leaves of Kratom and different times for boiling the Kratom tea (30 minutes or 1 hour) were compared to find the most proper conditions. Fresh leaves and a 1-hour boiling time were selected for preparing the tea. In detail, $40 \mathrm{~g}$ of fresh Kratom leaves were cut and mixed together with $2 \mathrm{~L}$ of distilled water, and the leaves were boiled for 1 hour. Three mitragynine concentrations of tea were prepared: $0.1042,0.166$, and $0.1917 \mathrm{mg} / \mathrm{mL}$. Then, the tea was kept in sterile $60 \mathrm{~mL}$ bottles and at $4^{\circ} \mathrm{C}$ for stability testing, which was performed at 3, 5, 7, and 14 days.

\section{Prestudy period}

On the first day, subjects were interviewed and underwent medical, physical, and basic psychological evaluations at Ramathibodi Hospital. The baseline mitragynine concentration and blood chemistry were also checked.

Since the subjects were chronic, regular users, and did not need to stop their Kratom use, we considered them adjusted to the steady state of Kratom levels by ingesting a known amount of Kratom tea (60 mL) every day for 7 days.

\section{Study period}

The pharmacokinetic study was performed on day 8. All subjects were admitted and resided in a ward for 24 hours. They were randomly assigned to take either $60 \mathrm{~mL}$ or $120 \mathrm{~mL}$ of the tea defined as loading doses or the 8th dose added in the study day. Forearm venous blood was collected from subjects through a heparin lock at 17 times points during the 24-hour period at time 0 (before the loading dose was given), 15, 30, and 45 minutes; $1,1.25,1.5,1.75,2,2.5,3,4,6,9,12,19$, and 24 hours. Urine samples were collected during the period of study to measure Kratom concentrations.

For physiologic changes and safety, vital signs as well as any abnormal signs and symptoms were also observed during the study period.

\section{Specimen analysis}

\section{Chemicals}

Mitragynine was purchased from Cerilliant Corporation (Round Rock, Texas, USA), 7-methyltestosterone from Fluka Chemie GmBH (Buchs, Switzerland), ethyl acetate from Thermo Fisher Scientific (Waltham, MA, USA), and acetic acid and methanol were from Merck KGaA (Darmstadt, Germany). All chemicals were of analytical grade.

\section{Analysis of plasma and urine samples}

A $0.5 \mathrm{~mL}$ aliquot of each subject's plasma and urine samples (indicate period of collection) was spiked with $25 \mu \mathrm{L}$ of $2.0 \mu \mathrm{g} / \mathrm{mL}$ 7-methyltestosterone (internal standard) to achieve final concentration $100 \mathrm{ng} / \mathrm{mL}$. A $2 \mathrm{~mL}$ aliquot of $0.1 \mathrm{M}$ phosphate buffer, $\mathrm{pH}$ 6.0, was added and briefly mixed. The solid phase extraction (SPE) column (C18; Agilent Technologies, Santa Clara, CA, USA) was conditioned with $2 \mathrm{~mL}$ of methanol and a $2 \mathrm{~mL}$ aliquot of $0.1 \mathrm{M}$ phosphate buffer, $\mathrm{pH}$ 6.0. The plasma or urine sample then was loaded onto a conditioned SPE column, and $1 \mathrm{~mL}$ of deionized water and $1 \mathrm{~mL}$ of $10 \mathrm{mM}$ acetic acid were added to the SPE column, which was dried for 5 minutes under vacuum $(<20 \mathrm{mmHg})$. Analytes were eluted with $3 \mathrm{~mL}$ of ethyl acetate and dried under a flow of nitrogen gas below $40^{\circ} \mathrm{C}$. This residue was reconstituted with $100 \mu \mathrm{L}$ of $20 \%$ acetonitrile.

\section{Analysis of Kratom tea}

A $10 \mu \mathrm{L}$ aliquot was added to $990 \mu \mathrm{L}$ of $20 \%$ (v/v) acetonitrile, mixed, and centrifuged at $13,800 \times g$ for 5 minutes at room temperature. The supernatant was used for subsequent analysis.

\section{Liquid chromatography-tandem mass spectrometry analysis}

The sample volume of $5 \mu \mathrm{L}$ (for plasma or urine) or $2 \mu \mathrm{L}$ (of Kratom tea) were analyzed using an Amazon SL LC-MS/ MS system. Liquid chromatography was performed in an UltiMate 3000 UHPLC system (Thermo Fisher Scientific) equipped with a Luna-C18 separation column $(100 \mathrm{~mm} \times$ $2.1 \mathrm{~mm} ; 3 \mu \mathrm{m}$ particle size) and a same phase guard column (10 $\mathrm{mm} \times 2.1 \mathrm{~mm} ; 3 \mu \mathrm{m}$ particle size). All samples were kept at $10^{\circ} \mathrm{C}$ in autosampler trays during the analysis cycle. Mobile phase A was composed of $5 \mathrm{mM}$ ammonium acetate together with $0.1 \%$ formic acid, and mobile phase $B$ was acetonitrile with $0.1 \%$ formic acid. After introduction of the sample, the mobile phase was initiated at 10\% B (90\% A) and linearly increased to $40 \% \mathrm{~B}$ within 10 minutes, then to $75 \% \mathrm{~B}$ within 13.5 minutes, and finally to $80 \% \mathrm{~B}$ within 16 minutes and maintained for 5 minutes.

Mass spectrometry was performed in an amaZon SL Ion Trap spectrometer (Bruker Daltonics, GmbH, Germany). A standard electrospray ionization source was operated in positive ion mode with the nebulizer at a gas flow rate of 8.0 L/minute and drying temperature of $250^{\circ} \mathrm{C}$. End plate offset and capillary voltage was $500 \mathrm{~V}$ and 4,500 V, 
respectively. Multiple reaction monitoring transition was $399.20 \rightarrow 238.20$ and $303.20 \rightarrow 285.20$ for mitragynine (retention time 9.10 minutes) and 7-methyltestosterone (retention time 13.74 minutes), respectively. Line spectra were collected in full scan mode from 50 to 1,000 Da. The validation of the analytical method was performed and will be published separately (Auparakkitanon, unpublished, 2015).

\section{Pharmacokinetics and statistical analysis}

The pharmacokinetics of mitragynine was evaluated by use of computer software, WinNonlin ${ }^{\circledR}$ version 3.0 (Pharsight Corporation, Mountain View, CA, USA). Noncompartmental analysis included determination of the following parameters: the maximum or peak plasma concentration $\left(\mathrm{C}_{\max }\right)$; the time to maximum observed concentration or time to reach $\mathrm{C}_{\max }\left(\mathrm{t}_{\max }\right)$; terminal half-life $\left(\mathrm{t}_{1 / 2}\right)$, estimated via linear regression of time and the log terminal end portion of the curve; and the area under the time-concentration curve from the time of dosing to the time of the last observable concentration $\left(\mathrm{AUC}_{0-\text { tlast }}\right)$, calculated by the log linear trapezoidal rule. The area under the curve (AUC) extrapolated to time to infinity $\left(\mathrm{AUC}_{0-\infty}\right)$ can be calculated by adding $\mathrm{AUC}_{0-\text { tlast }}$ with the last observable concentration $\left(\mathrm{C}_{\text {last }}\right)$ divided by linear regression-fitted terminal slope $\left(\lambda_{\mathrm{Z}}\right)$. The apparent total clearance (CL/F) was calculated by dose/ $\mathrm{AUC}_{0-\infty}$. The apparent volume of distribution $(\mathrm{Vd} / \mathrm{F})$ values were calculated in L/kg of body weight and were computed based on the terminal elimination phase and the apparent $\mathrm{CL} / \mathrm{F}$. Data were reported as the mean \pm standard deviation (SD) unless otherwise defined. Repeated measures ANOVA were tested for continuous data of mean blood pressure, pulse rate, and capillary blood glucose. The mean and SD were for continuous data, and the frequency and percentage were for categorical data.

\section{Results}

There were ten subjects recruited in this study. All were healthy men who had no preexisting diseases. The demographic data is shown in Table 1. The liver and kidney function values were within acceptable limit.

The data from our laboratory analysis showed that mitragynine concentration in the tea was stable during storage at the subjects' homes, and this meant that the subjects actually received the same dose every day.

The daily doses and loading doses were different and divided into five groups, as shown in Table 2. The normal and semilogarithmic plot of plasma mitragynine concentration
Table I The demographic data of ten subjects

\begin{tabular}{ll}
\hline Characteristics & Frequency \\
\hline Sex, Male $(\%)$ & $10(100)$ \\
Age (year), mean $\pm \mathrm{SD}$ & $27.1 \pm 4.7$ \\
Weight $(\mathrm{kg})$, mean $\pm \mathrm{SD}$ & $77.3 \pm 14.8$ \\
Height $(\mathrm{cm})$, mean $\pm \mathrm{SD}$ & $170.8 \pm 7.2$ \\
Body mass index $\left(\mathrm{kg} / \mathrm{m}^{2}\right)$, mean $\pm \mathrm{SD}$ & $26.4 \pm 3.9$ \\
Duration of abuse $($ year), median (min-max) & $\mathrm{I.75}(0.6-5)$ \\
Kratom leaves consumed/day, median (min-max) & $4(\mathrm{I}-9)$ \\
\hline Abbreviations: max, maximum; min, minimum; SD, standard deviation.
\end{tabular}

Abbreviations: max, maximum; min, minimum; SD, standard deviation.

against time curve of every subject after the administration of a loading dose are shown in Figure 1. We found the abnormal behavior of blood concentration in one subject, which was clearly far different from that of the other subjects. This subject's parameter was analyzed separately. The highest $\mathrm{C}_{\max }, 0.105 \mu \mathrm{g} / \mathrm{mL}$, and the highest $\mathrm{AUC}_{0-\text { tlast }}, 0.67 \mu \mathrm{g} \mathrm{h} / \mathrm{mL}$, were found in the subject taking the highest loading dose, $23 \mathrm{mg}$. The lowest $\mathrm{C}_{\max }$ was $0.0185 \mu \mathrm{g} / \mathrm{mL}$, found in the subject with the low loading dose $9.96 \mathrm{mg}$. The lowest $\mathrm{AUC}_{0 \text {-tlast }}$ was $0.062 \mu \mathrm{g} \mathrm{h} / \mathrm{mL}$, found in the subject with the lowest loading dose, $6.25 \mathrm{mg}$. The $\mathrm{T}_{\max }$ was $0.83 \pm 0.35$ hours and the average apparent $\mathrm{Vd} / \mathrm{F}$ and $\mathrm{CL} / \mathrm{F}$ of mitragynine were $38.04 \pm 24.32 \mathrm{~L} / \mathrm{kg}$ and $98.1 \pm 51.34 \mathrm{~L} / \mathrm{h} \mathrm{kg}$, respectively, as shown in Table 3. The semilog plot of pharmacokinetics time profiles in nine out of all ten subjects demonstrated biexponential decline, which suggested the pharmacokinetic behavior of mitragynine followed the oral two-compartment model, as shown in Figure 1. The correlation between the loading dose and $\mathrm{C}_{\max }$, as well as the loading dose and $\mathrm{AUC}_{0-\infty}$ were linear (Figures 2 and 3). When the dose increased, the $\mathrm{C}_{\max }$ and $\mathrm{AUC}_{0-\infty}$ increased proportionally with dose. We found that the $\mathrm{CL} / \mathrm{F}$ was quite constant and not increased when the loading dose increased. Altogether, it was suggested that the pharmacokinetics of mitragynine was linear or first order kinetics. The urine excretion of unchanged mitragynine was very limited, as it was only $0.14 \%$.

For one subject who took $9.96 \mathrm{mg}$ for both daily and loading doses and had the abnormal behavior of blood

Table 2 The number of subjects, the daily mitragynine doses to adjust for the steady state, and the loading doses in each subject

\begin{tabular}{lll}
\hline $\begin{array}{l}\text { Daily dose for } \\
\mathbf{7} \text { days }(\mathbf{m g} / \text { day) }\end{array}$ & $\begin{array}{l}\text { Loading dose }(\mathbf{m g}) \\
\text { in the study day (8th day) }\end{array}$ & $\begin{array}{l}\text { Number of } \\
\text { subjects }\end{array}$ \\
\hline 6.25 & 12.5 & 3 \\
6.25 & 6.25 & 2 \\
9.96 & 19.92 & 1 \\
9.96 & 9.96 & 3 \\
11.5 & 23 & 1 \\
\hline
\end{tabular}


A

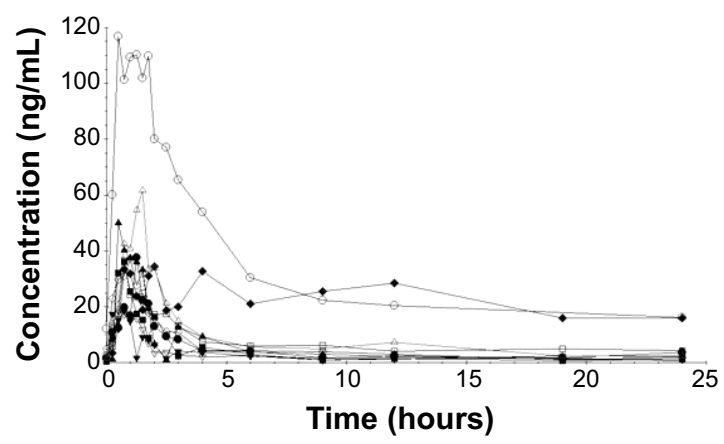

B

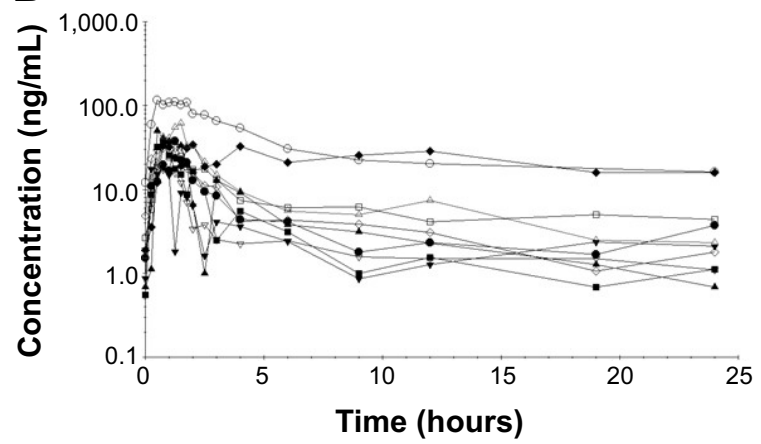

Figure I Plasma mitragynine concentration-time curve of every subject after the administration of a loading dose: the normal plot (A) and semi-logarithmic plot (B).

concentration, his normal and semilogarithmic plots are shown in Figure 4. There was no distribution phase because no sharp decrease of the blood concentration was seen. The possible errors were excluded by reevaluation. His blood samples were collected for other investigation in this regard.

The blood pressure and pulse rate of every subject increased at the 8th hour of study and then returned to normal, as shown in Figures 5 and 6. Capillary blood glucose levels were normal during the period of the study. All subjects described tongue numbness after they finished drinking Kratom tea. No abnormal signs and symptoms were detected during this period of the study.

\section{Discussion}

The pharmacokinetic parameters are important and essential information for the pharmacologic study of any substance of interest. To our knowledge, this study was the first pharmacokinetic study of Kratom in human. In our study, the pharmacokinetic parameters were different from those of animal studies. As we used different doses, more information was added, showing the pharmacokinetics were linear and followed an oral two-compartment model. Information on the bioavailability of oral mitragynine is not currently available in humans. The Vd and CL in our study were apparent Vd and CL. According to this apparent Vd, it would be

Table 3 The summary on the pharmacokinetic parameters of mitragynine

\begin{tabular}{ll}
\hline Parameters & Mean \pm SD \\
\hline $\mathrm{T}_{\max }(\mathrm{h})$ & $0.83 \pm 0.35$ \\
$\mathrm{Terminal}_{\mathrm{I} / 2}(\mathrm{~h})$ & $23.24 \pm 16.07$ \\
$\mathrm{Vd} / \mathrm{F}(\mathrm{L} / \mathrm{kg})$ & $38.04 \pm 24.32$ \\
$\mathrm{CL} / \mathrm{F}(\mathrm{L} / \mathrm{h} \mathrm{kg})$ & $98.1 \pm 51.34$ \\
\hline
\end{tabular}

Abbreviations: $\mathrm{CL} / \mathrm{F}$, clearance; $\mathrm{SD}$, standard deviation; $\mathrm{t}_{1 / 2}$, half-life; $\mathrm{T}_{\max }$, time to reach the maximum plasma concentration; $\mathrm{Vd} / \mathrm{F}$, volume of distribution; $\mathrm{h}$, hour. considered as a large $\mathrm{Vd}$, unless the bioavailability was less than $2 \%$. For example, we applied the oral bioavailability of mitragynine from the rat study, which was $3.03 \pm 1.47 \%{ }^{29}$ in our pharmacokinetic analysis. The estimated $\mathrm{Vd}$ and CL would be $1.15 \mathrm{~L} / \mathrm{kg}$ and $2.97 \mathrm{~L} / \mathrm{h} \mathrm{kg}$, respectively. We hypothesize that mitragynine has a large $\mathrm{Vd}$ and is mainly distributed out of the circulation.

As urine excretion of the unchanged mitragynine form was as low as $0.14 \%$, this suggests that renal excretion should not be a significant route of excretion, even when its bioavailability was taken into account. Based on our findings, we propose that mitragynine is mainly metabolized by hepatic metabolism to other metabolites. This is consistent with another study on mitragynine. ${ }^{39}$

As for the clinical and safety aspects, no serious adverse effect was found during the study. This is probably because the doses used in our study were less than $30 \mathrm{mg}$. However, the daily intake dose of regular abusers was reported to be as high as $276.5 \mathrm{mg}$ in Malaysia. ${ }^{16}$ Every subject in our study developed tongue numbness after having finished drinking Kratom tea. All of the participants also confirmed that it was the same experience as when they took Kratom. Thus, tongue

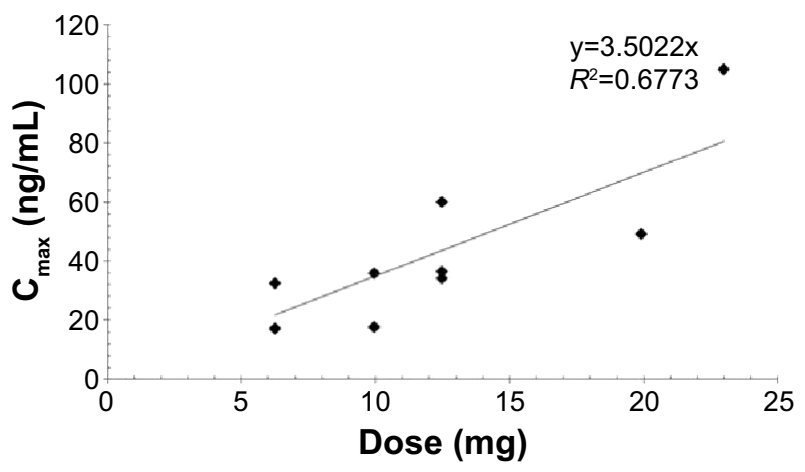

Figure 2 The relationship between the maximum plasma concentration $\left(\mathrm{C}_{\max }\right)$ and loading dose. 


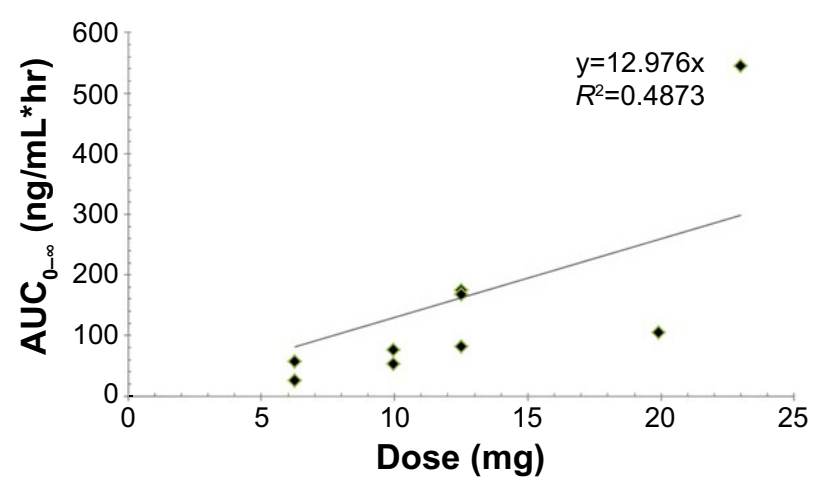

Figure 3 The relationship between the area under the curve extrapolated to time to infinity $\left(\mathrm{AUC}_{0-\infty}\right)$ and loading dose.

Abbreviation: hr, hour.

numbness would be one of the symptoms of Kratom effect that was supported by these studies. ${ }^{8,40}$

Our study also found that all subjects had increasing blood pressure and heart rate, but the onset was delayed to 8 hours after drinking Kratom tea. The time was later than $\mathrm{T}_{\max }$; therefore, this finding needs further investigation.
For clinical applications, the beneficial effects of Kratom on opioid withdrawal have been reported in various studies. Our study showed that it had a long elimination $\mathrm{t}_{1 / 2}$ and linear pharmacokinetics. Taken together, Kratom would be a good candidate for opioid substitute in patients who are addicted to these substances. As it had very low renal clearance, in patients with impaired renal function, dose adjustment might not be needed.

However, based on the finding that the pharmacokinetics of mitragynine is linear, if dose adjustment is needed, it can be easily implemented. Extracorporeal techniques such as hemodialysis or hemoperfusion might not be able to remove mitragynine from the circulation as it would have high $\mathrm{Vd} / \mathrm{F}$.

In conclusion, pharmacokinetics of mitragynine, a major and active metabolite of Kratom, is linear and follows the two-compartment model. It has a long terminal $\mathrm{t}_{1 / 2}$, extending for hours, and high apparent $\mathrm{Vd}$. Renal excretion of unchanged mitragynine is very low. The pharmacokinetics of Kratom in human was different from animals, thus applying the animal data to humans should be used with caution.
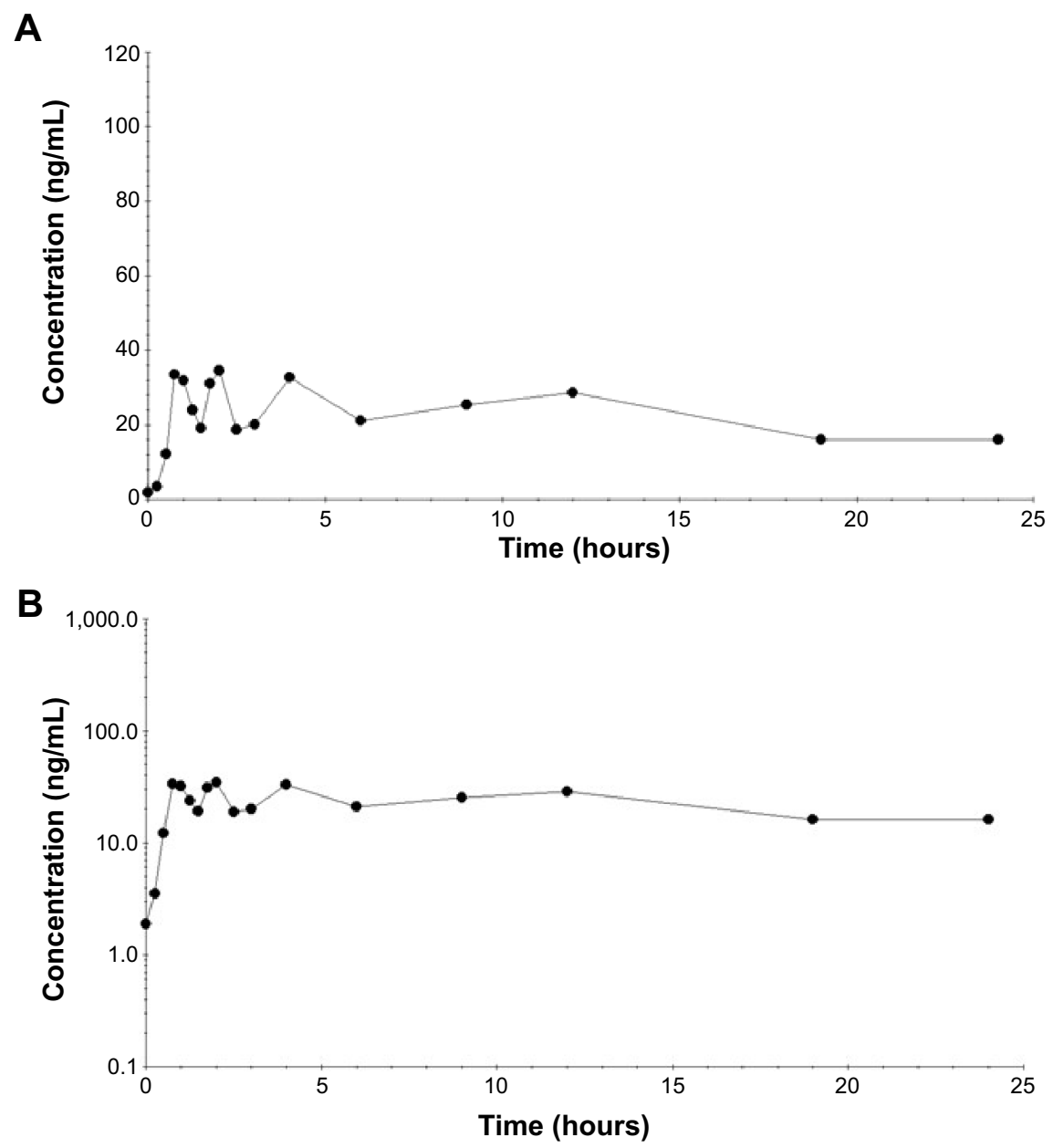

Figure 4 Plasma mitragynine concentration - time curve of one subject with abnormal behavior of blood concentration after the administration of a loading dose: the normal plot (A) and semi-logarithmic plot (B). 


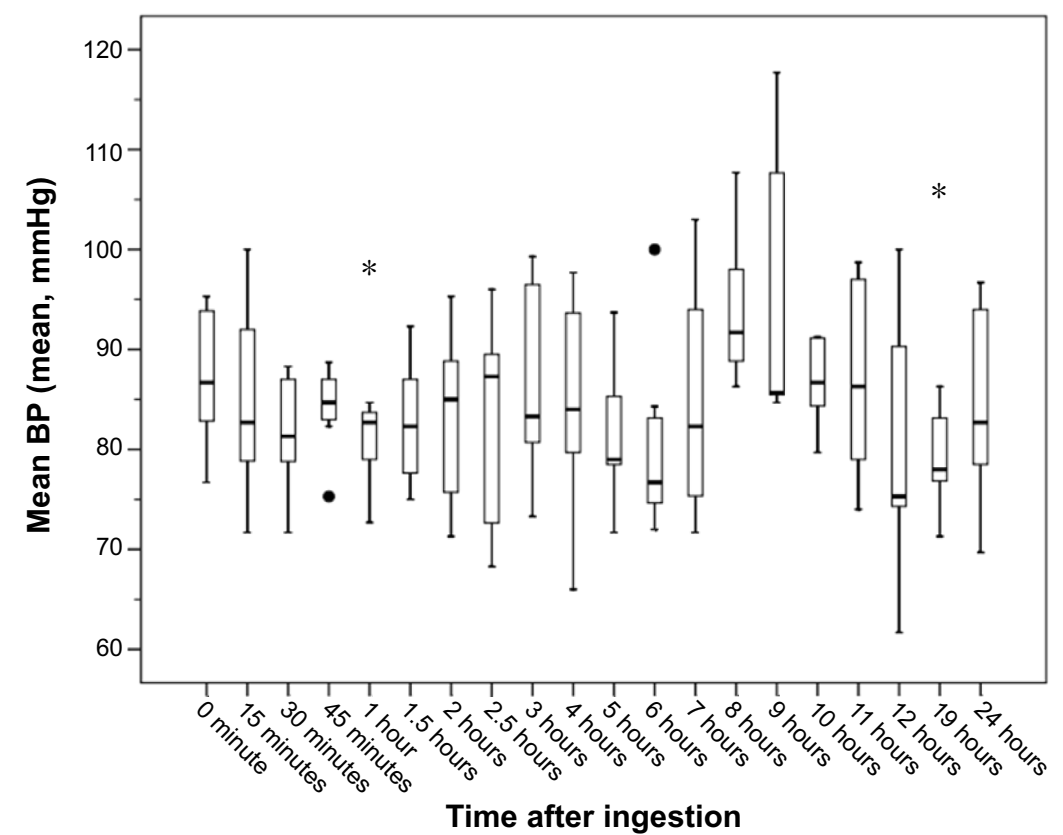

Figure 5 Blood pressures (BP) of every subject during the day of the study.

Notes: Upper fence represents maximum value, lower fence represents minimum value, the box represents the 25 th-75th percentile, the horizontal black line represents the 50 th percentile (median). ${ }^{*}$ Represents outlying value. *Represents extreme outlying value.

Numbness of tongue would be a clinical marker of Kratom ingestion. Abnormal pharmacokinetics in some patients and delayed onset of increasing blood pressure and heart rate require further investigation.

Kratom has been an emerging substance of abuse, available worldwide. In the future, Kratom may merit further scientific study to develop its medical benefits, as a better opioid substitute, with fewer lethal side effects, or as an effective pain killer.

\section{Limitation}

This study was done in chronic users. Therefore, the pharmacokinetic parameters would not apply for healthy subjects since the metabolism in chronic users might differ from that

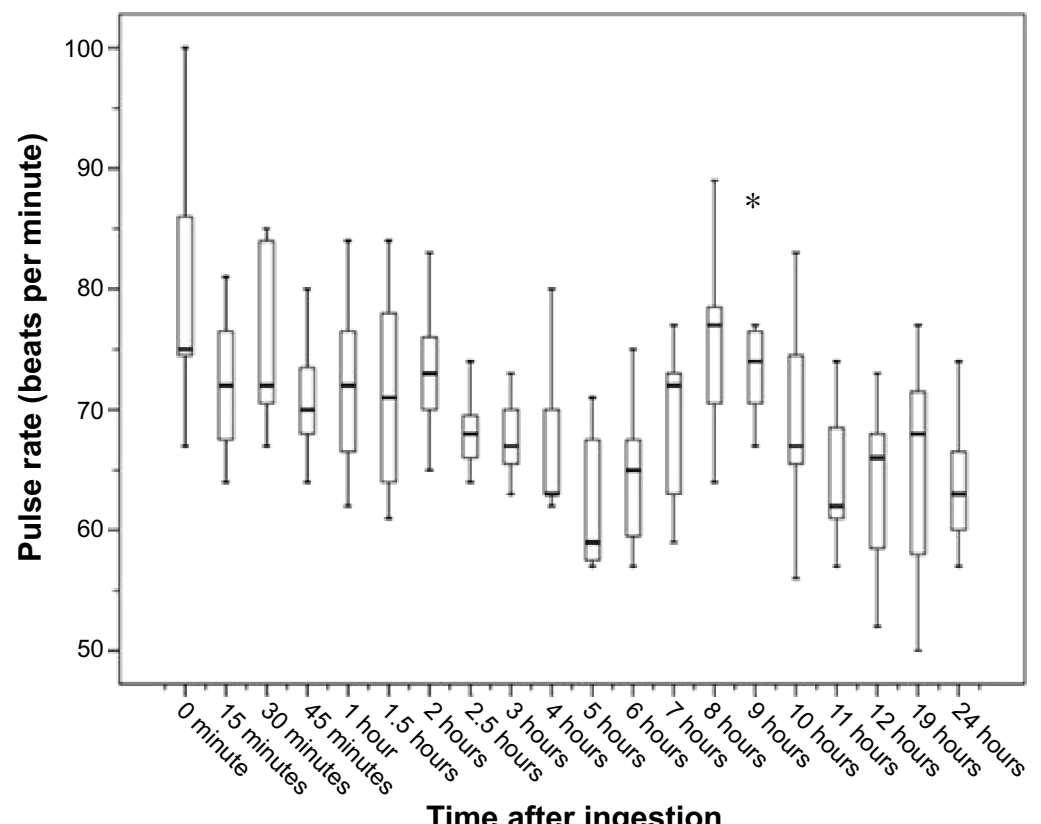

Figure 6 Pulse rates of every subject during the day of the study.

Notes: Upper fence represents maximum value, lower fence represents minimum value, the box represents the 25 th-75th percentile, the horizontal black line represents the 50 th percentile (median). *Represents extreme outlying value. 
of naïve persons. Further studies in healthy subjects and population pharmacokinetics should be carried out.

\section{Acknowledgments}

We wish to express our sincere thanks to Professor Amnuay Thithapandha for his help with English editing and comments on the manuscript and Mrs Umaporn Udomsubpayakul for the statistical analysis.

\section{Disclosure}

The authors report no conflicts of interest in this work.

\section{References}

1. Puff C, Chayamarit K, Chamchumroon V. Rubiaceae of Thailand: A Pictorial Guide to Indigeneous and Cultivated Genera. Bangkok: Forest Herbarium, National Park, Wildlife and Plant Conservation Department; 2005.

2. Hassan Z, Muzaimi M, Navaratnam V, et al. From Kratom to mitragynine and its derivatives: physiological and behavioural effects related to use, abuse, and addiction. Neurosci Biobehav Rev. 2013;37(2):138-151.

3. Suwanlert S. A study of kratom eaters in Thailand. Bull Narc. 1975;27(3):21-27.

4. Babu KM, McCurdy CR, Boyer EW. Opioid receptors and legal highs: Salvia divinorum and Kratom. Clin Toxicol (Phila). 2008;46(2): 146-152.

5. Boyer EW, Babu KM, Adkins JE, McCurdy CR, Halpern JH. Selftreatment of opioid withdrawal using kratom (Mitragynia speciosa korth). Addiction. 2008;103(6):1048-1050.

6. Boyer EW, Babu KM, Macalino GE, Compton W. Self-treatment of opioid withdrawal with a dietary supplement, Kratom. Am J Addict. 2007;16(5):352-356.

7. Vicknasingam B, Narayanan S, Beng GT, Mansor SM. The informal use of ketum (Mitragyna speciosa) for opioid withdrawal in the northern states of peninsular Malaysia and implications for drug substitution therapy. Int J Drug Policy. 2010;21(4):283-288.

8. Grewal KS. Observations on the pharmacology of mitragynine. J Pharmacol Expr Ther. 1932;46(3):251-271.

9. Macko E, Weisbach JA, Douglas B. Some observations on the pharmacology of mitragynine. Arch Int Pharmacodyn Ther. 1972;198(1): $145-161$.

10. Harizal SN, Mansor SM, Hasnan J, Tharakan JK, Abdullah J. Acute toxicity study of the standardized methanolic extract of Mitragyna speciosa Korth in rodent. J Ethnopharmacol. 2010;131(2): 404-409.

11. Kamal MSA, Ghazali AR, Yahya NA, Wasiman MI, Ismail Z. Acute toxicity study of standardized Mitragyna speciosa Korth aqueous extract in Sprague Dawley rats. J Plant Stud. 2012;1(2):120-129.

12. Ulbricht C, Costa D, Dao J, et al. An evidence-based systematic review of kratom (Mitragyna speciosa) by the Natural Standard Research Collaboration. J Diet Suppl. 2013;10(2):152-170.

13. Nelsen JL, Lapoint J, Hodgman MJ, Aldous KM. Seizure and coma following Kratom (Mitragynina speciosa Korth) exposure. J Med Toxicol. 2010;6(4):424-426.

14. Trakulsrichai S, Tongpo A, Sriapha C, et al. Kratom abuse in Ramathibodi Poison Center, Thailand: a five-year experience. J Psychoactive Drugs. 2013;45(5):404-408.

15. Sheleg SV, Collins GB. A coincidence of addiction to "Kratom" and severe primary hypothyroidism. J Addict Med. 2011;5(4):300-301.

16. Kapp FG, Maurer HH, Auwärter V, Winkelmann M, HermannsClausen M. Intrahepatic cholestasis following abuse of powdered kratom (Mitragyna speciosa). J Med Toxicol. 2011;7(3):227-231.
17. Apryani E, Hidayat MT, Moklas MA, Fakurazi S, Idayu NF. Effects of mitragynine from Mitragyna speciosa Korth leaves on working memory. J Ethnopharmacol. 2010;129(3):357-360.

18. McWhirter L, Morris S. A case report of inpatient detoxification after kratom (Mitragyna speciosa) dependence. Eur Addict Res. 2010;16(4): 229-231.

19. Singh D, Müller CP, Vicknasingam BK. Kratom (Mitragyna speciosa) dependence, withdrawal symptoms and craving in regular users. Drug Alcohol Depend. 2014;139:132-137.

20. Saingam D, Assanangkornchai S, Geater AF, Balthip Q. Pattern and consequences of krathom (Mitragyna speciosa Korth.) use among male villagers in southern Thailand: a qualitative study. Int J Drug Policy. 2013;24(4):351-358.

21. Saingam D, Assanangkornchai S, Geater AF, Lerkiatbundit S. Validation of Krathom (Mitragyna speciosa Korth.) Dependence Scale (KDS): a dependence screen for internationally emerging psychoactive substance. Subst Abus. 2014;35(3):276-283.

22. Kong WM, Chik Z, Ramachandra M, Subramaniam U, Aziddin RE, Mohamed Z. Evaluation of the effects of Mitragyna speciosa alkaloid extract on cytochrome P450 enzymes using a high throughput assay. Molecules. 2011;16(9):7344-7356.

23. Manda VK, Avula B, Ali Z, Khan IA, Walker LA, Khan SI. Evaluation of in vitro absorption, distribution, metabolism, and excretion (ADME) properties of mitragynine, 7-hydroxymitragynine, and mitraphylline. Planta Med. 2014;80(7):568-576.

24. Neerman MF, Frost RE, Deking J. A drug fatality involving Kratom. J Forensic Sci. 2013;58 Suppl 1:S278-S279.

25. Karinen R, Fosen JT, Rogde S, Vindenes V. An accidental poisoning with mitragynine. Forensic Sci Int. 2014;245C:e29-e32.

26. Ahmad K, AzizZ. Mitragyna speciosa use in the northern states of Malaysia: a cross-sectional study. J Ethnopharmacol. 2012;141(1):446-450.

27. Burillo-Putze G, López Briz E, Climent Díaz B, et al. [Emergent drugs (III): hallucinogenic plants and mushrooms]. An Sist Sanit Navar. 2013;36(3):505-518. Spanish.

28. Forrester MB. Kratom exposures reported to Texas poison centers. J Addict Dis. 2013;32(4):396-400.

29. Prozialeck WC, Jivan JK, Andurkar SV. Pharmacology of kratom: an emerging botanical agent with stimulant, analgesic and opioid-like effects. J Am Osteopath Assoc. 2012;112(12):792-799.

30. Rosenbaum CD, Carreiro SP, Babu KM. Here today, gone tomorrow... and back again? A review of herbal marijuana alternatives (K2, Spice), synthetic cathinones (bath salts), kratom, Salvia divinorum, methoxetamine, and piperazines. J Med Toxicol. 2012;8(1):15-32.

31. Schmidt MM, Sharma A, Schifano F, Feinmann C. "Legal highs" on the net-Evaluation of UK-based Websites, products and product information. Forensic Sci Int. 2011;206(1-3):92-97.

32. Ujváry I. Psychoactive natural products: overview of recent developments. Ann Ist Super Sanita. 2014;50(1):12-27.

33. Arndt T, Claussen U, Güssregen B, et al. Kratom alkaloids and O-desmethyltramadol in urine of a "Krypton" herbal mixture consumer. Forensic Sci Int. 2011;208(1-3):47-52.

34. Rech MA, Donahey E, Cappiello Dziedzic JM, Oh L, Greenhalgh E. New drugs of abuse. Pharmacotherapy. Epub 2014 Dec 4.

35. Ward J, Rosenbaum C, Hernon C, McCurdy CR, Boyer EW. Herbal medicines for the management of opioid addiction: safe and effective alternatives to conventional pharmacotherapy? CNS Drugs. 2011; 25(12):999-1007.

36. de Moraes NV, Moretti RA, Furr EB, McCurdy CR, Lanchote VL. Determination of mitragynine in rat plasma by LC-MS/MS: application to pharmacokinetics. J Chromatogr B Analyt Technol Biomed Life Sci. 2009;877(24):2593-2597.

37. Janchawee B, Keawpradub N, Chittrakarn S, Prasettho S, Wararatananurak P, Sawangjareon K. A high-performance liquid chromatographic method for determination of mitragynine in serum and its application to a pharmacokinetic study in rats. Biomed Chromatogr. 2007;21(2):176-183. 
38. Parthasarathy S, Ramanathan S, Ismail S, Adenan MI, Mansor SM, Murugaiyah V. Determination of mitragynine in plasma with solid-phase extraction and rapid HPLC-UV analysis, and its application to a pharmacokinetic study in rat. Anal Bioanal Chem. 2010;397(5):2023-2030.

39. Philipp AA, Wissenbach DK, Zoerntlein SW, Klein ON, Kanogsunthornrat J, Maurer HH. Studies on the metabolism of mitragynine, the main alkaloid of the herbal drug Kratom, in rat and human urine using liquid chromatography-linear ion trap mass spectrometry. J Mass Spectrom. 2009;44(8):1249-1261.
40. Chittrakarn S, Keawpradub N, Sawangjaroen K, Kansenalak S, Janchawee B. The neuromuscular blockade produced by pure alkaloid, mitragynine and methanol extract of kratom leaves (Mitragyna speciosa Korth.). J Ethnopharmacol. 2010;129(3):344-349.

\section{Publish your work in this journal}

Drug Design, Development and Therapy is an international, peerreviewed open-access journal that spans the spectrum of drug design and development through to clinical applications. Clinical outcomes, patient safety, and programs for the development and effective, safe, and sustained use of medicines are a feature of the journal, which has also been accepted for indexing on PubMed Central. The manuscript management system is completely online and includes a very quick and fair peer-review system, which is all easy to use. Visit http://www.dovepress.com/testimonials.php to read real quotes from published authors.

Submit your manuscript here: http://www.dovepress.com/drug-design-development-and-therapy-journal 